\title{
Replica Selection Algorithm for Streaming Media
}

\author{
Ge Yang* and Zhipeng Liu \\ Engineering Lab on Intelligent Perception for Internet of Things (ELIP), Shenzhen Graduate School, Peking University, Shenzhen \\ 518055, China \\ ${ }^{*}$ Corresponding author
}

\begin{abstract}
Replica selection algorithm for streaming media in Cloud-P2P (C2P2RSA2) is proposed in this paper. In replica selection model based on ant colony, a replica select metrics (replica node network bandwidth, network delay, etc.) is mapped by ant colony pheromone. The replica pheromone probability formula is proposed. The experimental results show the average access time of C2P2RSA2 is increased by $2 \%-5 \%$ than that of PARSA (pheromone-base ant colony replica adaptive selection algorithm in cloud storage) and best replica selection algorithm.
\end{abstract}

Keywords-Cloud-P2P; ant colony optimization; replica selection

\section{INTRODUCTION}

Because there are a large number of replicas of streaming media in a cloud environment and different replica selections cause different workloads on the virtual servers where the replicas reside, a replica selection policy of one number will make the load of the virtual server reach a more desirable value. In this paper, replica selection algorithm for streaming media in Cloud-P2P (C2P2RSA2) is proposed to choose the replica.

The Cloud-P2P storage structure in [1] consists of cloud service centers and P2P storage nodes. The cloud service center makes use of a large number of servers of streaming media providers, and users' requests are different at each different time, In some less busy time period, the virtual server can get a small amount of resources from the resource pool to be able to meet the user's request. During some busy periods, there are a large number of user requests. The virtual server can schedule a large amount of resources from the resource pool so that the processing capability of the virtual server in a short period of time is greatly improved and the user's request is responded to quickly. The virtual server can not only provide services to users, but also manage the storage nodes in $\mathrm{P} 2 \mathrm{P}$, view the online status of the nodes and monitor the load of the nodes and so on.

The current cloud storage system is mainly composed of GFS and HDFS with (Master / Slave) structure. [2] proposes a cloud storage system named Ming Cloud with equivalent cloud structure. By building a prototype system, Ming Cloud can implement data store, read, delete, search and other functions.

\section{replica selection algorithm}

In the streaming media system, in order to get better viewing quality, the system will copy multiple replicas, in a large number of replicas, which one is suitable for the user, so that users get better viewing quality, this is the problem of replica selection. In some cases, it is not the best replica node that has the shortest response time, but also depends on the needs of users. Therefore, a replica selection strategy for QoS preference perception is proposed, that is, QoS optimization. There are some bionic algorithms such as particle swarm optimization, genetic algorithms and more.

In the paper[3], it is proposed that in some cases, it is not the best replica node that has the shortest response time, but also depends on the needs of users. So, a replica selection strategy for QoS preference perception is proposed, that is, QoS optimization.

In the paper[4], according to reliability analysis of data nodes and network links, a reliability model of data replication service for cloud storage system is proposed. Based on the reliability of access and the number of replica between data, user access, reliability of data services designed, replication generation time and storage node selection method to determine the replica distribution and deletion algorithm. And conducted a series of experiments in ERS-Cloud of cloud storage system. The results show that the method can effectively protect the reliability of data services and further reduce the number of redundant storage.

Nowadays many replicas of data are placed on the cloud, so how to choose your replica on the cloud has also become a problem. [5] proposed a pheromone-base ant colony replica adaptive selection algorithm in cloud storage (PARSA). The PARSA algorithm is a selection of replicas stored on the cloud, if this algorithm is used in Cloud-P2P storage, because the cloud replica node is in a relatively good network environment, this will result in a large number of cloud copy nodes being used by users, which will increase operating costs for service providers, Therefore, the algorithm in this paper is optimized and modified so that the optimized algorithm can be used in Cloud-P2P. After the optimization, the replica node will give priority to the replica node in $\mathrm{P} 2 \mathrm{P}$. When the load factor of the P2P replica node reaches a certain level, the cloud copy node will be started by the user, allowing the user to get normal viewing quality.

\section{C2P2RSA2 ALGORITHM}

In this paper, the ant colony algorithm is optimized and the replica nodes on $\mathrm{P} 2 \mathrm{P}$ are prioritized to reduce the use of cloud replica nodes and reduce the cost of service providers.

(1) Initialize it with initialization formula, there are many parameters that affect the selection of replicas, such as the time the copy was read, the bandwidth of the network, network latency, the load completion rate of the replica node and 
whether it was a cloud replica node, and so on, using the initialization formula to make each copy have its own initial information prime value.

Pheromone Value: The initial pheromone value is initialized through the replica node's network environment and dynamically adjusted by the replica node's network environment and processing power.

$\operatorname{Td}_{a}^{j}(e)$ : Replica read time for replica node representing path e of user a to replica $j$, the replica's reading time at a moment $\mathrm{d}$, denoted as $\mathrm{w}_{\mathrm{d}}$, the biggest copy's reading time denoted as $\mathrm{w}_{\max }$.

$\operatorname{delay}_{a}^{j}(e)$ : Network delay for representing path e of user a to replica j, network latency in this article refers to network hops, the biggest network latency denoted as $\mathrm{m}_{\max }$, the smallest network latency denoted as $\mathrm{m}_{\min }$.

$b w_{a}^{j}(e)$ : Network broadband for representing path e of user a to replica $j$, Network bandwidth for replica path at a moment $d$, denoted as $n_{d}$.

$\operatorname{lr}_{a}^{j}(e)$ : Load completion rate for replica node representing path e of user a to replica $j$, the number of copies processed for replica node at moment $\mathrm{d}$ denoted as $\mathrm{y}_{\mathrm{d}}$, The total number of processing tasks for replica node denoted as $\mathrm{y}_{0}$

$$
\tau(0)_{j}=\frac{T d_{a}^{j}(e)}{f_{-} \text {size }}+\gamma_{j}
$$

Formula (1) Initialize for the replica j's pheromones,

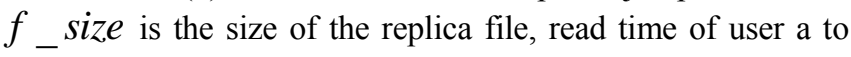
replica $\mathrm{j}$ denoted as $\operatorname{Td}_{a}^{j}(e)$, Initialize the pheromone value denoted as $\gamma_{j}$.

Formula (2) The initial total pheromone value for replica $\mathrm{j}$ consists of five parameters, which is:

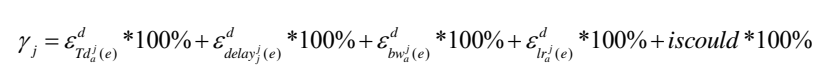

Several parameters in Formula (2) are respectively initialized by the following Formula (3), Formula 4),Formula (5), Formula (6).

$$
\begin{gathered}
\varepsilon_{T d_{a}^{j}(e)}^{d}=\left(w_{\max }-w_{d}\right) / w_{\max } \\
\varepsilon_{\text {delay }_{a}^{j}(e)}^{d}=\left(m_{\max }-m_{d}\right) / m_{\min }
\end{gathered}
$$

$$
\begin{gathered}
\varepsilon_{b w_{a}^{j}(e)}^{d}=\left(n_{\max }-n_{d}\right) / m_{\max } \\
\varepsilon_{l_{a}^{j}(e)}^{d}=y_{d} / y_{0}
\end{gathered}
$$

iscloud: Indicates whether it is a cloud replica node, this parameter increases the pheromone concentration of the cloud replica node if the load rate of the P2P replica node reaches a specified value, enable users to use cloud-replica nodes without degrading the quality of the user's viewing.

(2) Use Formula (7) to calculate the replica selection probability:

$$
p_{j}^{k}= \begin{cases}\frac{\left(\tau_{j}(t)\right)^{\alpha} \cdot\left(\vartheta_{j}\right)^{\beta}}{\sum_{j=1}^{q}\left(\tau_{j}(t)\right)^{\alpha} \cdot\left(\vartheta_{j}\right)^{\beta}} & j, q \in Z^{+} \\ 0 & \text { else }\end{cases}
$$

$\tau_{j}(t)$ : Represents the pheromone concentration of copy $\mathrm{j}$ at time $\mathrm{t}$.

$\vartheta_{j}$ : Represents the initial pheromone value of replica j.

$\alpha, \beta$ : Respectively, at the moment $t$, Indicates the importance of the concentration of pheromone and the importance of the initial pheromone value of the copy.

(3) Pheromone update, Formula (8) Is a copy of the successful choice or failure to choose is, pheromones will change, this will be modified by this formula. Formula (9) is when the copy is selected, the pheromone concentration of the copy is updated.

$$
\tau_{j}(t+1)=\rho^{*} \tau_{j}(t)+\tau_{j}
$$

$$
\tau_{j}(t+1)=\rho^{*} \tau_{j}(t)+(1-\rho) \bullet \varphi^{*} \tau_{j}
$$

$\tau_{j}$ : Expressed as the amount of pheromone change.

$\rho$ : Represents the residual coefficient of pheromone.

$\varphi$ :Expressed as the expand coefficient, this coefficient allows the pheromone to increase rapidly if there is a suitable replica.

The chart of the algorithm is:

(1) The first pheromone concentration initialization is performed for each replica node, can be based on the initial pheromone formula. 
(2) The probability of selection of each replica node is calculated according to the probability formula.

(3) Use the principle of probability matching, select the replica node.

(4) After a round of choices, some of the ants selected by the copy, using the reward formula to update its copy pheromone concentration, some not selected copy node, then use the penalty formula to update its copy pheromone concentration.

(5) Depending on the termination conditions, if the conditions are met, the result of the replica selection is output, if not, then return to step (2) to continue the next round of iteration.

\section{EXPERIMENTAL RESULTS AND ANALYSIS}

TABLE I. HARDWARE AND SOFTWARE PARAMETERS OF SIMULATION EXPERIMENT

\begin{tabular}{|c|c|c|c|c|c|c|}
\hline & $\begin{array}{c}\text { proce } \\
\text { ssor }\end{array}$ & RAM & $\begin{array}{c}\text { Operati } \\
\text { ng } \\
\text { system } \\
\text { median }\end{array}$ & $\begin{array}{c}\text { har } \\
\text { d } \\
\text { disk }\end{array}$ & $\begin{array}{c}\text { Progra } \\
\text { mming } \\
\text { software }\end{array}$ & $\begin{array}{c}\text { operating } \\
\text { system }\end{array}$ \\
\hline $\begin{array}{c}\text { param } \\
\text { eter }\end{array}$ & $\begin{array}{c}1.70 \mathrm{G} \\
\mathrm{Hz}\end{array}$ & $\begin{array}{c}8.00 \\
\mathrm{~GB}\end{array}$ & $64 \mathrm{bit}$ & $1 \mathrm{~T}$ & VS2012 & $\begin{array}{c}\text { Windows } \\
7\end{array}$ \\
\hline
\end{tabular}

Table 1 is the simulation environment used in this experiment, use Visual Studio 2012 Ultimate to generate a simulation environment, and the time, network latency, and network bandwidth for the replicas in the multiple replica nodes to be generated in the experiment are randomly generated, random numbers obey uniform distribution, uniform distribution means the occurrence of numbers in the range has the same probability.

This experiment analyzes the average access time of selecting replica nodes in Cloud-P2P network, comparisons with the PARSA algorithm are made with the best copy selection algorithm [1], concluded, the feasibility and advantages of $\mathrm{C}^{2} \mathrm{P}^{2} \mathrm{RSA} \mathrm{A}^{2}$ algorithm of this paper are proved, in this paper, the average access time, cloud copy node load rate as the evaluation criteria, repeat 3 for each experiment, take the average.

Figure 1 is when there are 10 replica nodes, comparison of the average access time of the text algorithm to that of the PARSA algorithm and the best replica selection algorithm, as can be seen from the figure when the replica node less, the average access time of the three algorithms are similar, however, the overall algorithm in this paper takes less time than the best copy selection algorithm, but $2.1 \%$ more time than the PARSA algorithm.

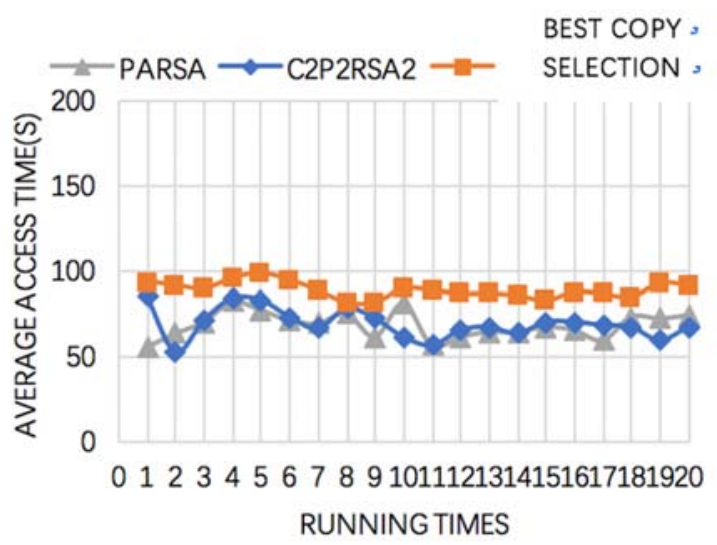

FIGURE I. AVERAGE ACCESS TIME COMPARING VARIOUS METHODS(10 REPLICA NODES).

\section{CONCLUSION}

The Cloud-P2P storage model we use in this paper is a combination of cloud-centric and P2P nodes to combine cloud flexibility, pay-as-you-go features and P2P scalability. This can be done by the flexibility of cloud computing. Since the ant colony algorithm to go through many iterations with the purpose of obtaining the best results, this calculation is very slow. This research will optimize the iterative process to reduce iterations and get the optimal solution quickly in the future work. Furthermore, the cloud replica nodes will be selectively reduced as the load factor of the cloud replica node reduced to a certain threshold, to reduce overhead on the cloud.

\section{ACKNOWLEDGMENT}

This research was financially supported by Major Scientific Research Project for Universities of Guangdong Province (201612008QX); Guangdong Provincial special funds Project for Discipline Construction (No.2013WYXM0122); Guangdong Provincial College Innovation and Entrepreneurship Project (201613177024); Scientific Research Project of Shenzhen (JCYJ20160428153620486, JCYJ20170303140803747); Key Laboratory of Intelligent Multimedia Technology(201762005).

\section{REFERENCES}

[1] Jin F, Wang C, Chen L, et al. Modeling and analysis of Cloud-P2P storage architecture[J]. Journal on Communications, 2015,03:33-40.

[2] Wu J, Fu J, Ping L, et al. Study on the P2P Cloud Storage System[J]. Acta Electronica Sinica,2011,v.39; No.33905:1100-1107.

[3] Zhao P, Huang T L, Liu C X, et al. Research of P2P architecture based on cloud computing[C]// Intelligent Computing and Integrated Systems (ICISS), 2010 International Conference on. IEEE, 2010:652-655.

[4] Xiong R, Luo J, Song A, et al. QoS preference-aware replica selection strategy in cloud computing[J]. Journal on Communications, 2011,07:93-102.

[5] CQ Huang, Y Li, HY Wu, et al. Modeling and maintaining the reliability of data replica service in cloud storage systems [J]. Journal on Communications, 2014,v.35;No.32210:89-97. 